\title{
De-noising Algorithm of Modified Thresholding Function Based On the Best Basis of Wavelet Packet
}

\author{
Chen Lin \\ Faculty of Information Engineering and Automation \\ KunmingUniversity of Science and Technology \\ Kunming,China \\ e-mail:sealinya@foxmail.com \\ Wang Wei \\ Faculty of Information Engineering and Automation \\ KunmingUniversity of Science and Technology \\ Kunming,China \\ e-mail:wangwei20032003@sina.com
}

\author{
Liu Zengli \\ Faculty of Information Engineering and Automation \\ KunmingUniversity of Science and Technology \\ Kunming,China \\ e-mail: liuzengli@hotmail.com \\ Tang Hui \\ Faculty of Information Engineering and Automation \\ KunmingUniversity of Science and Technology \\ Kunming,China \\ e-mail: thweibo@163.com
}

\begin{abstract}
This paper is about on denosing by modified thresholding function based on wavelet packet transform. First, there is a summary of the necessary conditions to construct the thresholding function on the basis of previous studies. And a new function for this method is put forward for wavelet packet denoising.Then,through four kinds of previous representative evolutionary thresholding functions are used for contrast, with the addition of traditional soft and hard threshoulding, all these functions are tested in a simulation experiment via four kinds of typical signals contarminated by different Gaussian white noise.Finally,the results show that the new function can be further remove noise effectively, and improve the signal-to-noise ratio, which reflects the superiority of conducting the modified thresholding function for wavelet packet signal denoising.
\end{abstract}

Keywords-wavelet packet, modified thresholding function, denoising, signal-to-noise ratio

\section{INTRODUCTION}

At present, both in theoretical research and engineering applications, the removal of signal interference with noise has been a hot topic. Although there are so many noise reduction algorithms, such as time composite method, frequency composite method, deconvolution, adaptive filtering,the cepstrum analysis method, artificial neural network and split spectrum analysis, the vast majority of them analyze signals only in the time or frequency domain.

As is well known, the wavelet analysis has been a new denoising method in recent years. Its excellent time-frequency local ability and good removed-correlation ability have been widely used in image compressionthe encoding field since 1989 when S.Mallat first introduced wavelet transform to image processing. And it has achieved good results[1]. Wavelet transform(WT) has multi-resolution characteristics as well as time-frequency localization. Because of different distribution characteristics in the wavelet coefficients at each scale between signal and noise, it can take the threshold method [2] to effectively achieve the goal of removing noise, especially processing non-stationary signals.With the development of technology, wavelet packet has more sophisticated analysis than wavelet,to breakdown the no subdivision of high-frequency portion in the wavelet transform.Therefore, we select wavelet packet to compensate for the deficiency of theWT, which only decomposes low frequency part of the signal.

Hard-threshold and soft-threshold function[2-3] are the most commonly used among threshold denoising algorithms. In hard threshold function,it is discontinuity that makes denoising incomplete , and noise still retain after denoising. While soft threshold function is fairly smooth, it is proved that there is constant deviation between the wavelet coefficients of the estimated signal and the true signal.If the noise signal is quite irregular, it may result in the fact that estimated signal is unusually smooth.To overcome these shortcomings, many experts and scholars at home and abroad have put forward all kinds of improved threshold function, but mostly for wavelet domain. Through analysis of previous four representative improved threshold functions,this paper gives a brief summary of the necessary conditions to conduct evolutional wavelet package denoising algorithms and a new one is presented for this method.Experimental results show that the new threshold function gives full play to the best wavelet packet basis threshold denoising , and this has important theoretical significance and application value at the same time.

\section{WAVELET PACKET}

In 1992, wavelet packet was named by Coifman, Meyer and Wickerhauser [4] at the foundation of wavelet transform. Wavelet packet transform(WPT) has fine resolution capability for signal analysis, for a better time-frequency localization characteristics and more adaptive ability to signal comparing with the wavelet transform. We have already known that wavelet analysis decomposes the signal into the rough part of low-frequency and the detail part of high-frequency, and then only does the same with low-frequency part ,for continuely breaking into the next low-frequency part and high-frequency part in 
multi-level segmentation, regardless of the high-frequency part.This only index interval divide for high-frequency part results in poor resolution at high frequencies, inevitably missing a lot of useful information in denoising, and seriously affecting the quality of the reconstructed signal.However, the wavelet packet analysis is further decomposing of the low-frequency part as well as the high-frequency part. As shown in figure 1 , where A is low frequency, D is high frequency, serial number at the end of letters represents wavelet decomposition layers. A signal by wavelet packet decomposition can be expressed as:

\section{$\mathrm{S}=\mathrm{AAA} 3+\mathrm{DAA} 3+\mathrm{ADA} 3+\mathrm{DDA} 3+\mathrm{AAD} 3+\mathrm{DAD} 3+\mathrm{ADD} 3+\mathrm{DDD} 3$}

As observed in Figure 1,the main advantage of wavelet packet analysis is to make high-frequency part of the D1,DA2,AD2,DD2 for segmentation.Wavelet packet analysis is a method of non-linear time-frequency area. According to the characteristics of signal, the method can adaptively select the appropriate frequency band to match the signal spectrum,and obviously it have improved the frequency resolution[5].Because of effective decomposition of a variety of time-varying signals, wavelet packet has a wider range of application value.

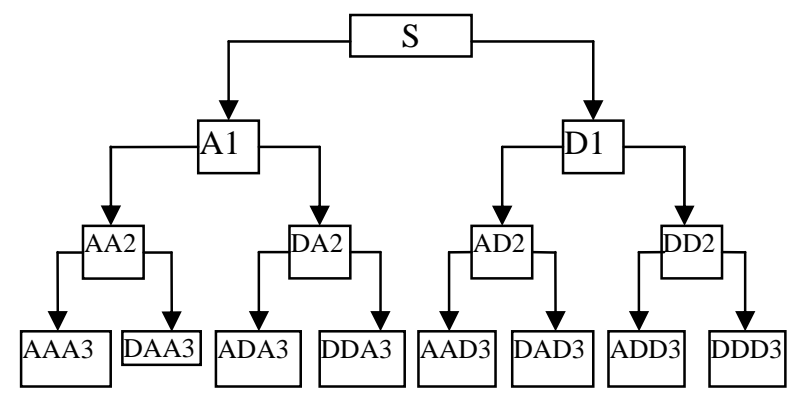

Figure1. Wavelet Packet Decomposition Tree Of Three Layers

The following is wavelet packet decomposition algorithm and reconstruction algorithm [6].

If the initial signal $g_{j}^{n}(t) \in U_{j}^{n}$, then $g_{j}^{n}$ can be expressed as:

$$
g_{j}^{n}(t)=\sum_{k} d_{k}^{j, n} u_{n}\left(2^{j} t-k\right)
$$

The algorithm for decomposition to the next subspace $U_{j+1}^{2 n}$ and $U_{j+1}^{2 n+1}$ is :

$$
\begin{aligned}
& d_{l}^{j+1,2 n}=\sum_{k} h_{k-2 l} d_{k}^{j, n} \\
& d_{l}^{j+1,2 n+1}=\sum_{k} g_{k-2 l} d_{k}^{j, n}
\end{aligned}
$$

The algorithm for Wavelet packet reconstruction:

$$
d_{k}^{j, n}=\sum_{l}\left(h_{k-2 l} d_{l}^{j+1,2 n}+g_{k-2 l} d_{l}^{j+1,2 n+1}\right)
$$

Where $d_{k}^{j, n}$ are coefficients of initial signal, $d_{l}^{j+1,2 n}$ and $d_{l}^{j+1,2 n+1}$ are coefficients of signal after decomposition, $h_{k-2 l}$ and $g_{k-2 l}$ are a pair of mirror filters.

\section{WAVELET PACKET BEST BASIS}

Wavelet packet library contains a wealth of wavelet packet basis. An intuitive way is to make use of all wavelet packet basis for noise reduction by WPT . In fact, different wavelet packet basis reflect the different signal characteristics [7-8] .What is also different based on different wavelet packet basis for the reconstruction of the same signal. we need to select a group of wavelet packet basis to represent the important features of the signal, removing those which are valueless .Obviously this is optimal wavelet packet basis if we pick out the best ones to characterize the nature of the signal as few as possible.

For example, after select the best wavelet packet basis, $\mathrm{S}$ from (1)can be simplified as:

$$
\mathrm{S}=\mathrm{AA} 2+\mathrm{ADA} 3+\mathrm{DDA} 3+\mathrm{D} 1
$$

In order to choose a group of better wavelet packet basis to describe the signal, first, we must define an information cost function to reflect the difference among sequence of the signal .It should take the smallest value of cost function from wavelet packet library for the best wavelet packetgroup. Coifman [9] and others put forward how to select the best basis based on the criterion "entropy", which was defined as cost function.The more different the sequence may be, the less entropy of the sequence should be.

A group of optimal wavelet packet basis could be decided by minimum entropy value of energy sequence through normalizing wavelet packet coefficients.It is to reflect average scattering properties of a whole set of coefficients on the basis of the criterion "entropy". Entropy is a universal concept in many applicated domains,especially in the field of signal processing. The entropy is an incremental information cost function, it must be meet:

$$
\left\{\begin{array}{l}
E(0)=0 \\
E(x)=\sum_{i} E\left(x_{i}\right)
\end{array}\right.
$$

Where $E$ is the entropy, $x$ is the signal, $x_{i}$ is the projection coefficients of the signal in an orthogonal wavelet packet base. The minimum entropy which requires $E(x)$ is the least.

Actully,the quick search way to select the optimal basis is to calculate from the bottom to the top and from right to 
left on the binary tree. The common entropy norms are Shannon entropy, the entropy of the norm, the entropy of the threshold, SURE entropy and logarithmic energy entropy. Shannon entropy is defined as follows:

$$
E\left(x_{i}\right)=x_{i}^{2} \log \left(x_{i}^{2}\right)
$$

\section{WAVELET PACKET DENOISING}

\section{A. Denoising Principle}

In practical applications, the useful signal is usually low-frequency stationary signal, while the noise is usually high-frequency signal. Set up a one-dimensional noisy signal model:

$$
f(t)=s(t)+n(t)
$$

Where $s(t)$ is original signal with no superimposed noise, $n(t)$ is Gaussian white noise with variance for 1 , and it obeys $N\left(0, \sigma^{2}\right)$. If we want to recover the original signal $s(t)$ from $f(t)$, it is necessary to suppress noise. Wavelet shrinkage [10]shows that the wavelet coefficients $w_{j, k}$ still are composed of two parts after discrete wavelet transform. One is the wavelet coefficients $u_{j, k}$ corresponding with the signal $s(k), k \in z$, and the other part is the wavelet coefficients $v_{j, k}$ corresponding with noise $n(k), k \in z$.

$$
w_{j, k}=u_{j, k}+v_{j, k}(j, k \in z)
$$

The basic idea of threshold denoising which is proposed by Donoho and Johnstone [2,3] is below:

For hard-thresholding,the method may discard the part of wavelet coefficients ${ }^{w_{j, k}}$ if they are less than a critical threshold $t$ as the fused wavelet coefficients $w_{j, k}$ are mainly caused by noise. Otherwise, they are mainly caused by signal, and should be retained.

For soft-thresholding, the fused wavelet coefficients

$w_{j, k}$ may shrink to zero by a fixed amount if they are more than a critical threshold $t$. After processing as the above solutions, the new fused wavelet coefficients are implemented by wavelet inverse transform in order to realize the separation of signal and noise.

Similarly, the wavelet packet coefficients of noisy signal are linearly superposed by the signal and noise after wavelet packet transform.WP analysis is quite sensitive to the singular point of the signal as well as Gaussian white noise that is singular everywhere.So to some extent discontinuous point will appear in the regained signal and wavelet packet coefficients will also mutate under the influence of the white noise.Therefore, the signal and noise have distinct characteristics of wavelet packet transform.Through Selecting the best wavelet packet basis to represent the important features of the signal for threshold denoising,this not only greatly optimizes the calculation, but also specifically improves the denoising ability.

Following are four steps in wavelet packet shrinkage method :

a) Apply the discrete wavelet packet transform (WPT) to signal $f(t)$ and obtain the empirical wavelet coefficients $w_{j, k}(j, k \in z)$ at scale $j$. Note to select a suitable wavelet base function and decomposition scale $j$.

b) Select the appropriate standard of entropy to compute the optimal tree of the wavelet packet.

c) Apply the nonlinearity,such as hard-thresholding function, $\bar{W}_{j, k}=w_{j, k},\left|w_{j, k}\right| \geq t$ to the empirical wavelet packet coefficients at each scale $j$.Then the estimate coefficients $\bar{W}_{j, k}$ are obtained based on the unified threshold $t=\sigma \sqrt{2 \lg n}, n=$ length $(f(k))$. Generally, in order to get optimal results to meet the specific analysis and evaluation criteria, the thresholding function should be determined through several repeated experiments to make $\left|\bar{W}_{j, k}-u_{j, k}\right|$ as small as possible.

d). Use the inverse WPT on thresholded wavelet packet coefficients.

In the above four steps, the key is how to select thresholding value and the thresholding operations.It is directly related to the quality of denoising to some extent.

The wavelet packet shrinkage method relies on the basic idea that the energy of a function (with some smoothness) will often be concentrated in a few coefficients in wavelet packet domain while the energy of noise spreads among all coefficients in wavelet packet domain. Therefore, the nonlinear thresholding function in wavelet domain will tend to keep a few of larger coefficients representing the function, while the noise coefficients will tend to reduce to zero.

\section{B. Improved Thresholding Function}

Even though both hard thresholding and soft thresholding have been widely applicated in practice and also achieved certain effect, the algorithms themselves have some defects [10]. In order to overcome the shortcomings in hard and soft thresholding,it is so advisable to combine two methods to build a new threshold function.Nowadays, many research scholars at home and abroad have put forward various improved threshold functions.In the paper four typical threshold functions are selected for comparative study.

(1)Root mean square(RMS) method[11]

$$
\bar{W}_{j, k}= \begin{cases}\operatorname{sgn}\left(w_{j, k}\right) \sqrt{\left(w_{j, k}^{2}+\left(w_{j, k}-t\right)^{2}\right) / 2} & ,\left|w_{j, k}\right| \geq t \\ 0 & ,\left|w_{j, k}\right|<t\end{cases}
$$


Where $t$ is threshold value, $w_{j, k}$ are coefficients of signal after decomposition by wavelet paceket, $\bar{W}_{j, k}$ are coefficients of signal after processing by threshold function. It is easy to prove that $\sqrt{\left(w_{j, k}^{2}+\left(w_{j, k}-t\right)^{2}\right) / 2}$ is always bewteen $\left|w_{j, k}\right|$ and $\left|w_{j, k}\right|-t$,then reduces constant deviation t bewteen the estimated coefficients $\bar{W}_{j, k}$ and $w_{j, k}$. However, the function is discontinuous at $\mathrm{t}$.

(2) compromis method[12]

$$
\bar{W}_{j, k}= \begin{cases}\left.\operatorname{sgn}\left(w_{j, k}\right)\left|w_{j, k}\right|-\alpha * t\right] & ,\left|w_{j, k}\right| \geq t \\ 0 & ,\left|w_{j, k}\right|<t\end{cases}
$$

Where $\alpha \in[0,1]$, the value of $\alpha$ is adjustable, which depends on denoising effect. When $\alpha=0$, the function is hard threshold function. When $\alpha \neq 0$, the function is soft threshold function. However, the function is discontinuous at $\mathrm{t}$ when $\alpha \neq 0$.

(3)elimination method[13]

$$
\bar{W}_{j, k}= \begin{cases}\operatorname{sgn}\left(w_{j, k}\right)\left[\left|w_{j, k}\right|-\frac{t}{1+\ln \left(\left|w_{j, k}\right|-t+1\right)}\right],\left|w_{j, k}\right| \geq t \\ 0 \quad,\left|w_{j, k}\right|<t\end{cases}
$$

This function makes use of elimination method. Along with the increase of $\left|w_{j, k}\right|, \bar{W}_{j, k}$ gradually tends to $\left|w_{j, k}\right|$, avoiding the error.Meanwhile, the function is continuous at t.

(4)index method[14]

$$
\bar{W}_{j, k}= \begin{cases}\operatorname{sgn}\left(w_{j, k}\right)\left[\left|w_{j, k}\right|-\frac{2 t}{1+\exp \left(\left(\left|w_{j, k}\right|-t\right)^{2} / N\right)}\right],\left|w_{j, k}\right| \geq t \\ 0 \quad,\left|w_{j, k}\right|<t\end{cases}
$$

Where $\mathrm{N}>0,{ }_{t}=\frac{\sigma \sqrt{2 \lg N}}{\lg (j+1)}$. Based on the formula 13, it is continuous. When $\left|w_{j, k}\right|>t$, it is high-level derivable [14]. In fact,the adjustment ability of the function is not so flexible because of $\mathrm{N}$ as on-denominator regulatory factor.In order to achieve better noise reduction than traditional thresholding, empirical value of $\mathrm{N}$ actually tends to be smaller.The function is increasing with $\left.\right|_{w_{j, k}}$ add.It is easy to

cause the function to approach hard-threshold function,making it difficult to solve the singularity problems in restoration of the signal effectively.

We can summarize some rules from the above four improved threshold function
If a function is monotone nondecreasing,as $\bar{W}_{j, k}=f\left(w_{j, k}\right)$,it must satisfy two essential condtions as forllows.

a)As $w_{j, k}$ gradually increases, $\bar{W}_{j, k}$ must gradually approach to $w_{j, k}$.

b)As $w_{j, k}$ gradually tends to $t, \bar{W}_{j, k}$ must gradually be approximate 0 or $t$.

\section{(5)weighting method}

According to the above conditions, the paper constructs a new threshold function under the summary of the advantages and disadvantages of the improved threshold function.

$$
\bar{W}_{j, k}= \begin{cases}(1-\beta) w_{j, k}+\beta \operatorname{sgn}\left(w_{j, k}\right)\left[\left|w_{j, k}\right|-\frac{2 t}{1+\sqrt{\beta}}\right],\left|w_{j, k}\right| \geq \mathrm{t} \\ 0 & ,\left|w_{j, k}\right|<t\end{cases}
$$

Where $\beta$ is variable, $\beta=\exp \left(-\alpha\left(\left|w_{j, k}\right|-t\right)^{2}\right)$, and $\alpha>0$.The new function has the following advantages:

Firstly, the new one still has continuity.

If $\left|w_{j, k}\right|=t, \bar{W}_{j, k}=0$. If $\left|w_{j, k}\right| \rightarrow t, \bar{W}_{j, k} \rightarrow 0$.

So the function is continuous when $\left|w_{j, k}\right|=t$ and high-level derivative when $\left|w_{j, k}\right|>t$. It is just needed for various mathematical processing conveniently, to avoid the deficiency of hard threshold function.

Secondly, when $w_{j, k}>0, \frac{\bar{W}_{j, k}}{w_{j, k}}=1-\frac{2 t * \beta}{w_{j, k}(1+\sqrt{\beta})}$. the case is equivalent to that when $w_{j, k} \rightarrow+\infty, \quad \beta \rightarrow 0$ and $\overline{w_{j, k}} \rightarrow w_{j, k}$. Similarly, when $w_{j, k} \rightarrow-\infty, \overline{w_{j, k}} \rightarrow w_{j, k}$. Then, the new function takes the straight line $\overline{w_{j, k}}=w_{j, k}$ as the asymptote, along with $w_{j, k}$ increasing, $\bar{W}_{j, k}$ gradually approaches to $w_{j, k}$, avioding constant deviation between $\bar{W}_{j, k}$ and $w_{j, k}$ in soft-threshold function.

At last,the new one overcomes the shortcoming of the hard thresholding with discontinuous function and solves the problem of the permanent bias in soft thresholding. Futhermore, the new function is more flexible and more extensive by changing an adjustment factor $\alpha$. As long as regulating the value of $\alpha$ can we get more applicable threshold function to achieve the purpose of denoising effectively.

\section{SIMULATION AND ANALYSIS}

In order to test noise elimination of the five above improved threshold functions based on wavelet packet transform and illustrate the validity of the new one, four kinds of test signals Blocks,Bumps, Heavy sine, Doppler contarminated with white Gaussian noise were selected to 
simulate denoising from a Matlab toolbox, also compared with experiments of traditional soft-threshold and hard-threshold under wavelet packet denoising. By trial-and-error, to reach the best denoising effect, the paper used in the simulation with compactly supported rthogonal wavelet function $\mathrm{db} 4$ of high vanishing moments and 4-layer decomposition. The value of threshold is $t=\sigma \sqrt{2 \lg n}$, with $n$ expressing the length of signal.It is shannon-Weaker entropy that adopted the best wavelet packet basis in this paper. Since there are regulatory factors in the functions, we select the best denoising results as a reference after repeated adjustments. Figure 2 shows Heavysine signal with snr = 20.4057 after denoising by six ways except soft-thresholding.

The paper introduces the evaluation index with signal-to-noise ratio (SNR) and mean square error (MSE) for quantitative analysis so as to accurately compare the denoising effect.The expressions as follows:

$$
\begin{gathered}
M S E=\frac{1}{N} \sum_{i=1}^{N}\left(y_{i}-\overline{y_{i}}\right)^{2} \\
S N R=10 \lg \frac{\sum_{i=1}^{N} y_{i}^{2}}{\sum_{i=1}^{N}\left(y_{i}-\overline{y_{i}}\right)^{2}}
\end{gathered}
$$

Where $\mathrm{N}$ is the data length, $y_{i}$ is the original noise-free signal, $\overline{y_{i}}$ is the signal after noise reduction. They show the comparative data of SNR and MSE through seven denoising ways in different noise conditions in Table 1 and Table 2.

Through subjective analysis in figure 2, it shows diagrams besides soft-thresholding whose distortion is the most serious after denoising.Hard-thresholding does notdenoise completely, and the image obviously contains a lot of burrs.Root mean square(RMS) method and compromise method apply discontinuous functions, we can see that singular points are presented in detail place of images. Elimination, index and weighted methods effectively overcome the above shortcomings for which the smooth functions are utilized, so as to restore original signal better. Hence in the same condition, the continuous functions are superior to the discontinuous ones in denoising. Among the whole reconstructed images in figure 2, the one obtained by weighting method not only effectively removes noise interference, but also looks more smooth, with it clearly recovering the shape of the original signal.
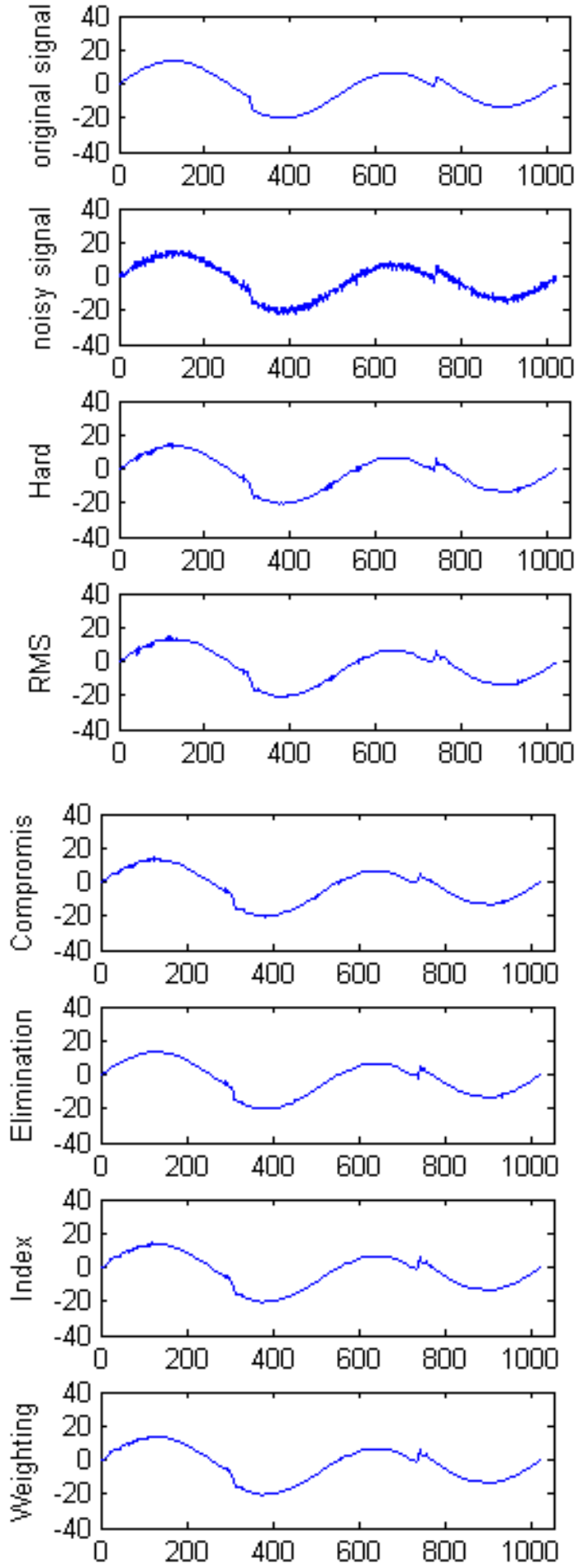

Figure 2 denoised Heavysine signals by six ways

Through objective analysis in Table 1 and Table 2, the higher SNR and the smaller MSE may be, the stronger denoising capability should have. We used seven ways to display denoising data with four kinds of noisy signal.Compared with the traditional soft-thresholding,it can 
be seen that these modified thresholding functions have all improved the SNR at different degrees. RMS and compromise methods were inferior in denising in comparison with hard-thresholding. This indicates smoothness property of the estimate using improved thresholding functions have affected denoising ability,which is necessary to consider first for constructing threshold funtions.More-over,these methods of elimination , index and weighting significantly denoise better than the other ones, because of adjustable parameters exist in the index and the weighting methods.That makes the denoising more flexible.As long as changing the parameters value, you can maintain the higher signal-to-noise ratio.

In this paper, the new one- weighting method improves the signal-to-noise ratio and reduces the mean square error, significantly displaying to be superior to the other methods

TABLEI. SNR FOR DIFFERENT THREHOLDING SCHEMES

\begin{tabular}{|c|c|c|c|c|c|c|c|c|}
\hline Ways & \multicolumn{8}{|c|}{ Four Kinds Of Signals } \\
\hline Signals & Doppler & Heavysine & Bumps & Blocks & Doppler & Heavysine & Bumps & Blocks \\
\hline NoisySignals & 14.1707 & 14.3851 & 15.4095 & 17.5693 & 20.1913 & 20.4057 & 21.4301 & 23.5899 \\
\hline Soft & 17.1523 & 17.6516 & 18.4482 & 17.9166 & 21.9107 & 23.0624 & 23.1197 & 22.7635 \\
\hline Hard & 21.2951 & 21.7750 & 21.2322 & 22.6832 & 26.6446 & 27.6953 & 27.3677 & 28.2274 \\
\hline RMS & 18.9371 & 20.5564 & 19.8933 & 20.6093 & 24.0741 & 26.0208 & 25.7286 & 25.9285 \\
\hline Compromis & 20.2264 & 21.0727 & 21.0693 & 21.4800 & 25.7501 & 26.7082 & 26.4080 & 26.4840 \\
\hline Elimination & 21.5262 & 22.7466 & 21.7210 & 22.1524 & 27.2524 & 28.2039 & 26.9690 & 27.5449 \\
\hline Index & 21.8999 & 22.7473 & 22.0234 & 22.8412 & 27.7577 & 28.6232 & 27.5493 & 28.5693 \\
\hline Weighting & 22.1259 & 23.6986 & 22.1937 & 22.9209 & 27.7704 & 28.8598 & 27.7610 & 28.6596 \\
\hline
\end{tabular}

TABLEII. MSE RISK FOR DIFFERENT THREHOLDING SCHEMES

\begin{tabular}{|c|c|c|c|c|c|c|c|c|}
\hline \multirow{2}{*}{$\begin{array}{c}\text { Ways } \\
\text { Signals }\end{array}$} & \multicolumn{8}{|c|}{ Four Kinds Of Signals } \\
\hline & Doppler & Heavysine & Bumps & Blocks & Doppler & Heavysine & Bumps & Blocks \\
\hline NoisySignals & 0.9827 & 0.9827 & 0.9827 & 0.9827 & 0.9827 & 0.9827 & 0.9827 & 0.9827 \\
\hline Soft & 0.5670 & 0.4632 & 0.4882 & 0.9072 & 0.6614 & 0.5330 & 0.6660 & 1.1887 \\
\hline Hard & 0.1905 & 0.1793 & 0.2571 & 0.3027 & 0.2224 & 0.1834 & 0.2504 & 0.3378 \\
\hline RMS & 0.3279 & 0.2373 & 0.3500 & 0.4880 & 0.4019 & 0.2697 & 0.3652 & 0.5735 \\
\hline compromis & 0.2437 & 0.2107 & 0.2653 & 0.3994 & 0.2732 & 0.2302 & 0.3124 & 0.5037 \\
\hline Elimination & 0.1762 & 0.1433 & 0.2298 & 0.3421 & 0.1933 & 0.1632 & 0.2745 & 0.3953 \\
\hline Index & 0.1608 & 0.1406 & 0.2143 & 0.2919 & 0.2049 & 0.1440 & 0.2402 & 0.3155 \\
\hline Weighting & 0.1574 & 0.1151 & 0.2061 & 0.2869 & 0.2032 & 0.1408 & 0.2287 & 0.2129 \\
\hline
\end{tabular}

practical advice . Moreover,I thank my friends who give

\section{CONCLUSION}

On the one hand, in the paper a new modified thresholding function is proposed by comparative analysis of the four kinds of typical improved threshold functions.And the introduction of optimal wavelet packet basis has broken the traditional wavelet shrinkage.On the other hand, with the simulation which uses four signals in matlab toolbox under different noisy conditions to compare the effect of noise elimination,it can be shown that the new threshold function further improve the signal-to-noise ratio and reduce the mean square error.The significance of the new one is its fine continuous derivative and flexible adjustment. As long as changing the parameters, we can obtain the ideal denoising effect according to the actual signal.As a result, the new thresholding function gives full play to the superiority of the optimal basis of wavelet packet to denoise.This has obvious advantages and broad application prospects.

\section{ACKNOWLEDGMENT}

I sincerely express my gratitude to my tutor for his careful instruction and strong support.I am also gratfull all partners in the laboratory for their enthusiastic help and assistance for my translating. With all their valuable opinions,I was able to finish the paper.

This work was supported by Natural Science Foundation Project of NSFC under the grant No.60872157 and No.61271007

\section{REFERENCES}

[1] S.Mallat,Z.Zhang, "Matching pursuit with time-frequency dictionaries," IEEE Transacions on Signal Processing,vol.41, pp. 3397-3415, Dec 1993.

[2] D.L.Donoho and I.M.Johnstone, "Ideal spatial adapation by waveletshrinkage,”Biometrika,vol.81,pp.425-455.Mar 1994.

[3] D.L.Donoho, "De-noising by soft-thresholding,"IEEE Trans. Inform. Theroy,vol.41,pp.613-627,Mar 1995

[4] G.S.Hu,“Modern signal processing”,China,tsinghua university press, 2005

[5] I.Daubechies, “Ten Lectures on Wavelets, ”China, National Defense Industry Press, 2004

[6] D.F.Zhang,“MATLAB wavelet analysis (2),"Beijing,China Machine Press, Dec.2011

[7] M.S.Reis,P.M.Saraiva andB.R.Bakshi, "Multiscale statistical process control using wavelet packets,”AICHE Journal, vol.54, pp. 2366-2378,Sep 2008 
[8] C.F.Zhang and L.J.Zhu, "Voice signal compression method based on wavelet packet best basis," Journal of Data Acquisition and Processing,vol.25,pp.744-750,2010.

[9] R.R.Coifman, M.V.Wickerhauser, "Entropy-based algorithms for best basis selection,” IEEE Trans.on Information Theroy, 1992. 238(3):713-718

[10] W.Q.Zhang and G.X.Song, "signal de-noising in wavelet based on a new kind of thresholding function," China, Journal of Xidian University, Vol.31,pp.297-299, Apr.2004.

[11] K.R.Jiang,X.Q.Tang,D.Q.Zhu, “Automotive wheel speed signal processing based on the improved wavelet thresholding algorithm" Chinese Journal of Scientific Instrument, Vol.31.pp. 736- 740,2010.
[12] Y.Q.Wang and L.X.Wei,“Analysis on modified wavelet threshold de-noising functions, "China,J.University of Shanghai for Science and Technology, Vol.33,pp.405-408, April 2011

[13] S.Z.Wang and Y.T.Zhu, "Comparative Simulation Study of Evolutional Wavelet Based on Denoising Algorithms,” Noise And Vibration Control,Vol.32,pp.128-132,Jan.2012

[14] X.Q.Jin and J.B.Wang, "Application of improved denoising algorithm of wavelet packet threshold in guided wave detection signal processing,” Foreign Electronic Measurement Technology, Vol.31,pp.55-58,June 2012

[15] H.Y.Tao,H.F.Qin and C.B.Yu."Reseach of Signal Denoising Method Based on an Improved Wavelet Threaholding,". PIEZOEL ECTRICS and ACOUSTOOPTICS, Vol.30 , pp.93-95, Jan. 2008 